\title{
Aberrations analysis of a Focused Plenoptic Camera
}

\section{Alessandro Grosso, Kurt Vonmetz, Toralf Scharf}

Alessandro Grosso, Kurt Vonmetz, Toralf Scharf, "Aberrations analysis of a Focused Plenoptic Camera ," Proc. SPIE 10677, Unconventional Optical Imaging, 106772T (24 May 2018); doi: 10.1117/12.2306860 


\title{
Aberrations analysis of a Focused Plenoptic Camera
}

\author{
Alessandro Grosso*ab, Kurt Vonmetz ${ }^{\mathrm{a}}$, Toralf Scharf ${ }^{\mathrm{b}}$ \\ ${ }^{a}$ Datalogic IP Tech Srl, Via San Vitalino, 13, 40012, Lippo, Calderara di Reno, Italy; \\ ${ }^{b}$ Nanophotonics and Metrology Laboratory (NAM), EPFL, Station 11, CH 1015, Lausanne, \\ Switzerland
}

\begin{abstract}
A Focused Plenoptic Camera in Galilean configuration is studied and its aberrations behavior is interpreted with the Nodal Aberration Theory (NAT). Sequential ray tracing is applied to individual optical channels constituted by the camera objective and individual decentered microlenses. The wavefront aberration field is retrieved at the exit pupil of the optical channels and is analyzed through the Zernike Fringe decomposition technique. Decentered optical channels show nodes in the field-dependence of different Zernike coefficients approximating the wavefront aberration field. The nodal behavior is a consequence of the loss of rotational symmetry in a decentered optical channel due to the displacement of a microlens with respect to the mechanical axis of the camera.
\end{abstract}

Keywords: Focused Plenoptic Camera, Microlens Array, Multi-Aperture optical system, Nodal Aberration Theory, Zernike Fringe Polynomials

\section{INTRODUCTION}

A plenoptic camera is a multi-aperture scaled optical system based on the addition of a microlens array (MLA) between the objective and the image sensor of a standard camera. The MLA is a 2-dimensional array of refractive lenses with diameters typically ranging from $0.1 \mathrm{~mm}$ to $1 \mathrm{~mm}$. The sequence of image sensor and MLA constitutes a plenoptic sensor. There are two main variants of plenoptic camera: the "unfocused" Plenoptic Camera or 1.0 and the "focused" Plenoptic Camera or 2.0. In 2005 Ren $\mathrm{Ng}^{1}$ implemented the Plenoptic Camera 1.0. The main features of the 1.0 variant are the "refocusability" over a larger depth of field compared to a standard camera with similar optical parameters and a faster image acquisition speed due to the use of an objective with a larger aperture (lower F-number) and a better SNR.

In 2009 Georgiev and Lumsdaine ${ }^{2}$ proposed the Plenoptic Camera 2.0. In 2012 Perwass ${ }^{3}$ realized a multi-focus plenoptic camera based on a MLA with multiple focal lengths. The main difference in construction between the 1.0 and the 2.0 camera is the distance between the image sensor and the MLA: in the former case such a distance is equal to the microlens focal length, in the latter case such a distance is larger or smaller than the microlens focal length. The multifocus plenoptic camera is an example of Focused Plenoptic Camera where the MLA is characterized by multiple focal lengths.

The Focused Plenoptic Camera can be distinguished in two different sub-variants: the "real image" or Keplerian Plenoptic Camera and the "virtual image" or Galilean Plenoptic Camera. In the Keplerian camera, the main objective creates a real intermediate image in front of the MLA that relays it into the image sensor. On the contrary, in the Galilean camera the main objective creates a virtual image behind the plenoptic sensor. The distance between the image sensor and the MLA needs to be adjusted properly in order to realize a Keplerian or a Galilean configuration.

*alessandro.grosso@datalogic.com, alessandro.grosso@epfl.ch 
In this paper, a Focused Plenoptic Camera in Galilean configuration is studied. Figure 1 shows the configuration in detail. The camera main objective is a Cooke Triplet with $12^{\circ}$ Field of View (FOV) followed by MLA made of fused silica ( $\mathrm{n}=1.458$ ) with $40 \times 40$ circular refractive microlenses arranged in a quadratic grid with $0.25 \mathrm{~mm}$ pitch (equal to the microlens diameter) and Radius of curvature (ROC) equal to $0.489 \mathrm{~mm}$. Because of the multi-aperture nature of a Plenoptic Camera, sequential ray tracing technique cannot be applied to the overall system but only to the analysis of individual channels of the camera. A single channel is defined by the Cooke Triplet followed by individual microlens and is identified by the microlens location in the 2D array. The optical axis of the overall system coincides with its mechanical axis connecting the vertex of the optical surfaces constituting the Cooke Triplet with the vertex of the central microlens in the MLA with $(0,0)$ coordinates. The mechanical axis of the camera is the reference axis for the whole optical system. The $(0,0)$ microlens is the only axial channel with rotational symmetry about the optical axis of the camera, while all the others are displaced from the mechanical axis and constitute decentered channels. A decentered channel loses the rotational symmetry about the optical axis of the system originating a particular behavior of the aberrations different from that expected from a rotationally symmetric system. In what follows we will discuss the monochromatic $(\lambda=0.55 \mu \mathrm{m})$ aberrations properties of such a configuration with particular interest on the effect of decentering.

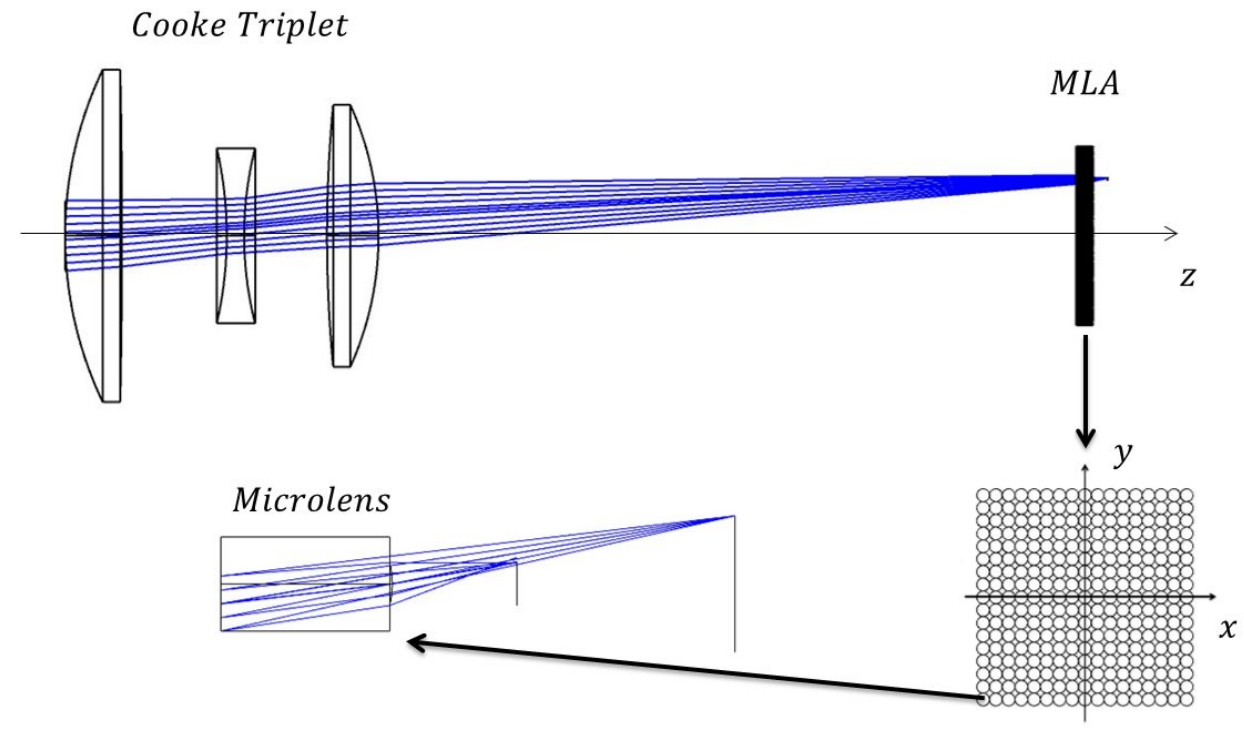

Figure 1: Layout of a Focused Plenoptic Camera in Galilean configuration.

\section{PARAXIAL EQUIVALENT OF A DECENTERED OPTICAL CHANNEL}

For each channel the light rays are collected by the main objective into a sub-area of the image sensor, thus creating a microimage. A decentered microlens intercepts the light rays coming from an off-axis region of the Cooke Triplet FOV. The position of a microimage on the image sensor is determined by the corresponding decentered microlens forming it. The microimage height needs to be equal to the microlens diameter in order to use the whole area of the image sensor and avoid overlapping or vignetted images. Thus a raw plenoptic image is formed by a 2D array of microimages replicating the MLA geometry.

The channels of a Focused Plenoptic Camera can be described by the coordinates of the corresponding microlens in the MLA plane. The microlens in the origin of the MLA plane constitutes the only on-axis channel with rotational symmetry and its reference axis coincides with the mechanical axis of the camera while the other microlenses constitute off-axis channels identified by their decentering coordinates measured from the mechanical axis of the camera. 
For decentered channels the optical axis is a real ray starting at the center of the object plane, passing through the center of the pupils for all the optical surfaces in the channel and locating the center of the Gaussian image at the image plane $\vec{H}=H_{x} \vec{\imath}+H_{y} \vec{\jmath}$ : this real ray is defined as Optical Axis Ray (OAR). The OAR allows the definition of an equivalent paraxial system for a decentered channel because it determines the position of its entrance and exit pupils. A decentered microlens in an optical channel gives its contribution to the total aberration field along a direction passing by its center of curvature $C$ and by the intersection between its exit pupil $\vec{\rho}=\rho_{x} \vec{\imath}+\rho_{y} \vec{\jmath}$ with the OAR. The position of $C$ in the Image Plane measured from the OAR is a vector $\vec{\sigma}=\sigma_{x} \vec{\imath}+\sigma_{y} \vec{\jmath}$ representing the displacement of the wavefront aberration field contribution of a decentered microlens as illustrated in Figure 2.

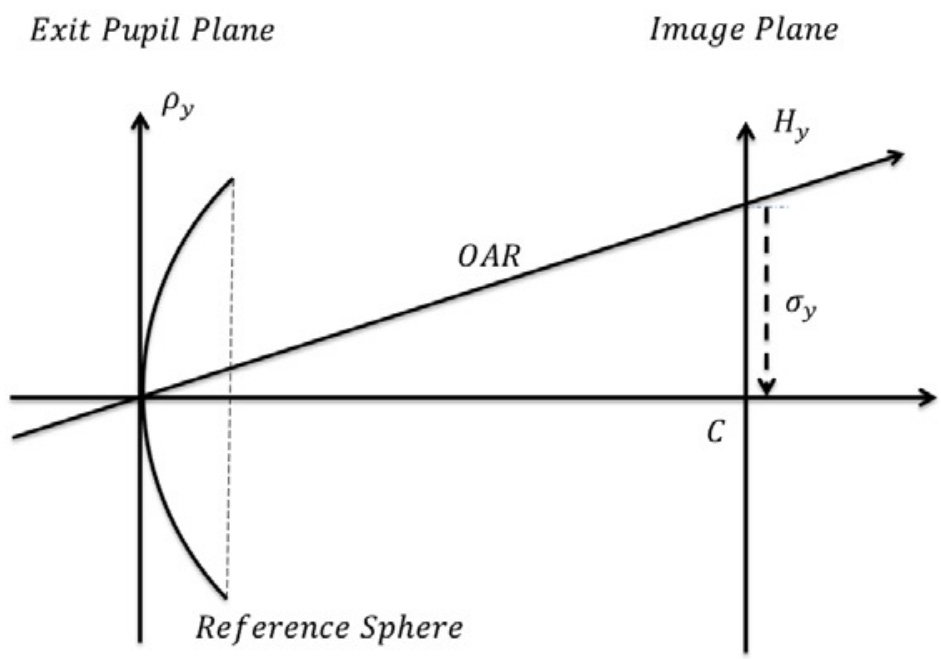

Figure 2: The OAR passes through the center of the Exit Pupil of a decentered channel and its intersection with the Gaussian Image Plane defines the image displacement. The reference Sphere at the exit Pupil of the optical channel contributes to the total aberration field along a direction passing through the center of the Exit pupil (located by the OAR) and its Center of

Curvature C.

\section{WAVEFRONT ABERRATION FIELD FOR DECENTERED OPTICAL CHANNELS}

As proposed by Shack ${ }^{4}$ and Thompson ${ }^{5}$, a decentered optical system with no rotational symmetry can be studied in the framework of nodal aberration theory (NAT) that is an extension of the Hopkins wave aberration theory ${ }^{6}$ to optical systems without rotational symmetry. In NAT the wave aberration field $W$ is expressed in vectorial form with the following expression

$$
W=W[(\vec{H} \cdot \vec{H}),(\vec{H} \cdot \vec{\rho}),(\vec{\rho} \cdot \vec{\rho})]=\sum_{j} \sum_{p}^{\infty} \sum_{n}^{\infty} \sum_{m}^{\infty}\left(W_{k l m}\right)_{j}(\vec{H} \cdot \vec{H})^{p}(\vec{\rho} \cdot \vec{\rho})^{n}(\vec{H} \cdot \vec{\rho})^{m}
$$

where $\vec{H}$ is the field vector measured as image height, $\vec{\rho}$ indicates the point of intersection of a light ray with the exit pupil, $W_{k l m}$ are the aberration coefficients for every surface $j$ in an optical channel.

Adding the contribution of decentered microlenses through the displacement vector $\vec{\sigma}$, the resulting wave aberration field in vectorial form can be expressed as 


$$
\begin{gathered}
W=W[((\vec{H}-\vec{\sigma}) \cdot(\vec{H}-\vec{\sigma})),((\vec{H}-\vec{\sigma}) \cdot \vec{\rho}),(\vec{\rho} \cdot \vec{\rho})]= \\
=\sum_{j} \sum_{p}^{\infty} \sum_{n}^{\infty} \sum_{m}^{\infty}\left(W_{k l m}\right)_{j}\left(\left(\vec{H}-\overrightarrow{\sigma_{J}}\right) \cdot\left(\vec{H}-\overrightarrow{\sigma_{J}}\right)\right)^{p}(\vec{\rho} \cdot \vec{\rho})^{n}\left(\left(\vec{H}-\overrightarrow{\sigma_{J}}\right) \cdot \vec{\rho}\right)^{m}
\end{gathered}
$$

$\vec{\sigma}$ enables the definition of an effective field vector $\vec{H}_{e f f}$ as

$$
\vec{H}_{e f f}=\vec{H}-\vec{\sigma}
$$

that accounts for the geometrical perturbation of an optical channel deriving from its decentered microlens as illustrated in Figure 3.

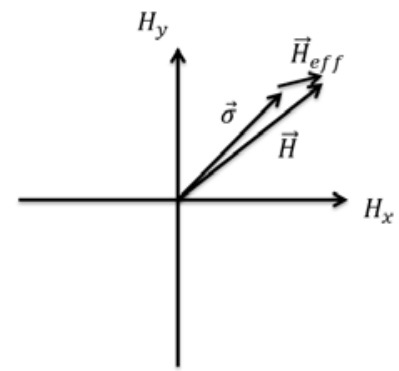

Figure 3: Effective field in the image plane for a decentered channel.

The total aberrated field is the sum of the contributions from all the optical surfaces constituting a channel. The contribution due to a decentered microlens is centered along a direction intersecting the image plane at a point depending on the vector $\vec{\sigma}$. The final aberration function exhibits a field-dependent nodal behavior due to the definition of a new effective field deriving from the decentered geometry of the optical channels.

\section{ANALYSIS OF MULTICHANNEL IMAGING SYSTEM WITH DECENTERED CHANNELS}

As an example, we will consider the channel identified by the microlens with $(-0.25 \mathrm{~mm}, 0.25 \mathrm{~mm})$ decentered coordinates measured from the mechanical axis of the system, whose layout is shown in Figure 4 and we will compare its wavefront error with that of the on-axis channel.

\section{Cooke Triplet}

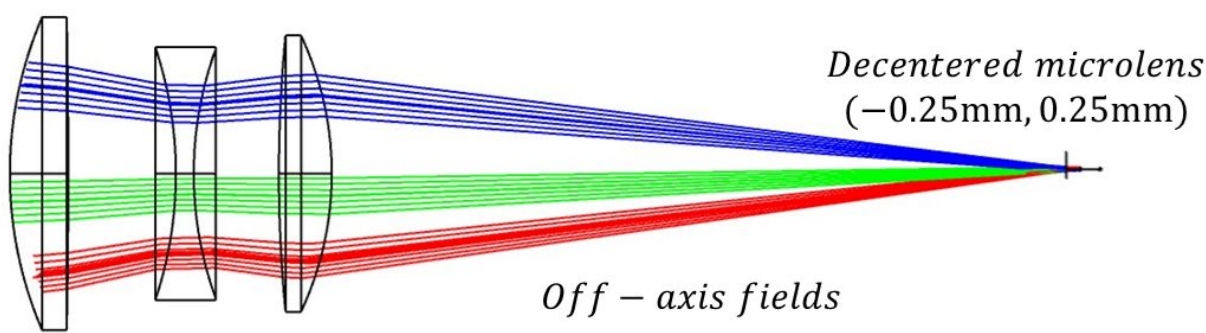

Figure 4: $(-0.25 \mathrm{~mm}, 0.25 \mathrm{~mm})$ decentered channel layout. 

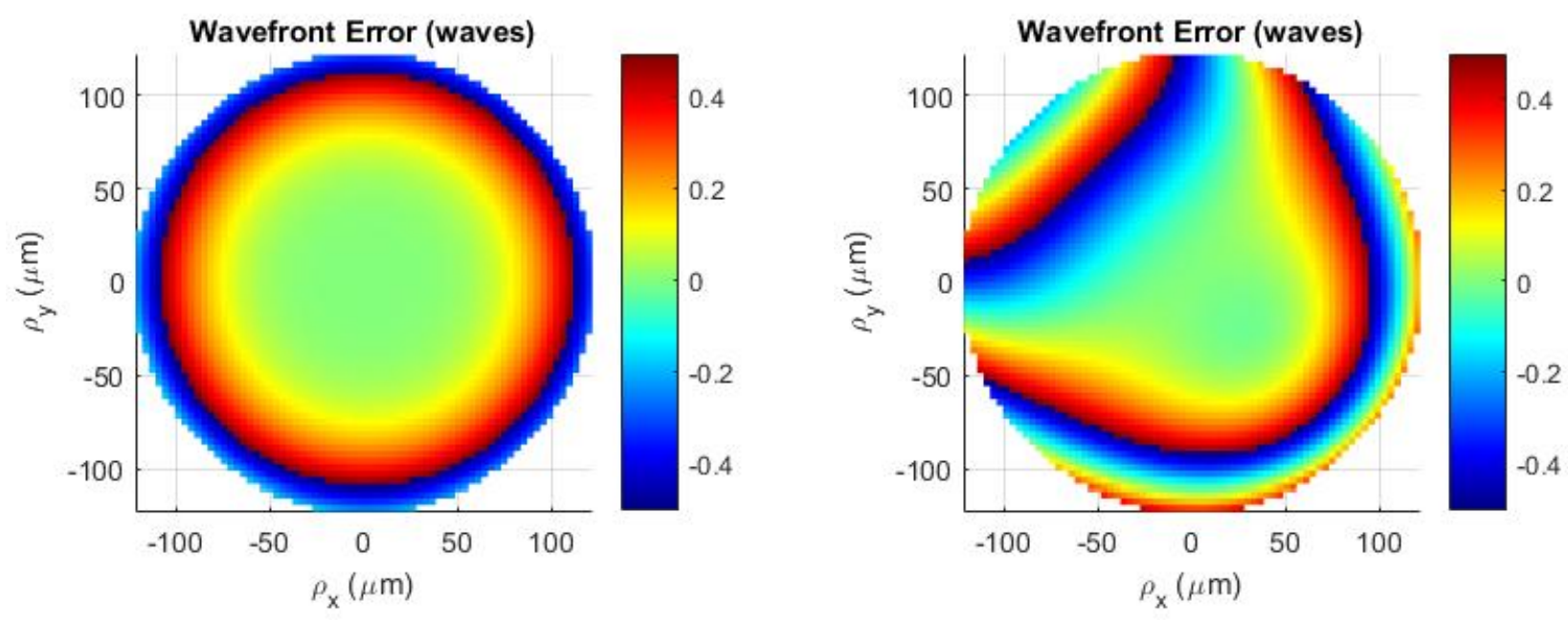

Figure 5: Left: Wavefront aberration field at the exit pupil of the on-axis channel for on-axis field point $\left(\boldsymbol{H}_{x}=\mathbf{0}, \boldsymbol{H}_{\boldsymbol{y}}=\mathbf{0}\right)$. Right: Wavefront aberration field at the exit pupil of the on-axis channel for the normalized field point $\left(H_{x}=-0.707\right.$, $\left.H_{y}=0.707\right)$.

Figure 5 shows the wavefront error of the central channel of the Focused Plenoptic Camera for an on-axis field point $\left(H_{x}=0, H_{y}=0\right)$ and for an off-axis field point with normalized coordinates $\left(H_{x}=-0.707, H_{y}=0.707\right)$, respectively.

When $\left(H_{x}=0, H_{y}=0\right)$ is applied, the wavefront error related to the on-axis channel is rotational symmetric about the mechanical axis of the camera passing through the center of the Exit Pupil $\left(\rho_{x}=0, \rho_{y}=0\right)$ as is evident from the graph on the left in Figure 5. Defocus and spherical aberration are the main contributions to it. When an off-axis field point ( $\left.H_{x}=-0.707, H_{y}=0.707\right)$ is applied to the on-axis channel, additional aberration contributions are summed at the Exit Pupil of the system such as coma and astigmatism and the final wavefront map is perturbed along the direction of the applied field as is evident from the graph on the right in Figure 5. 

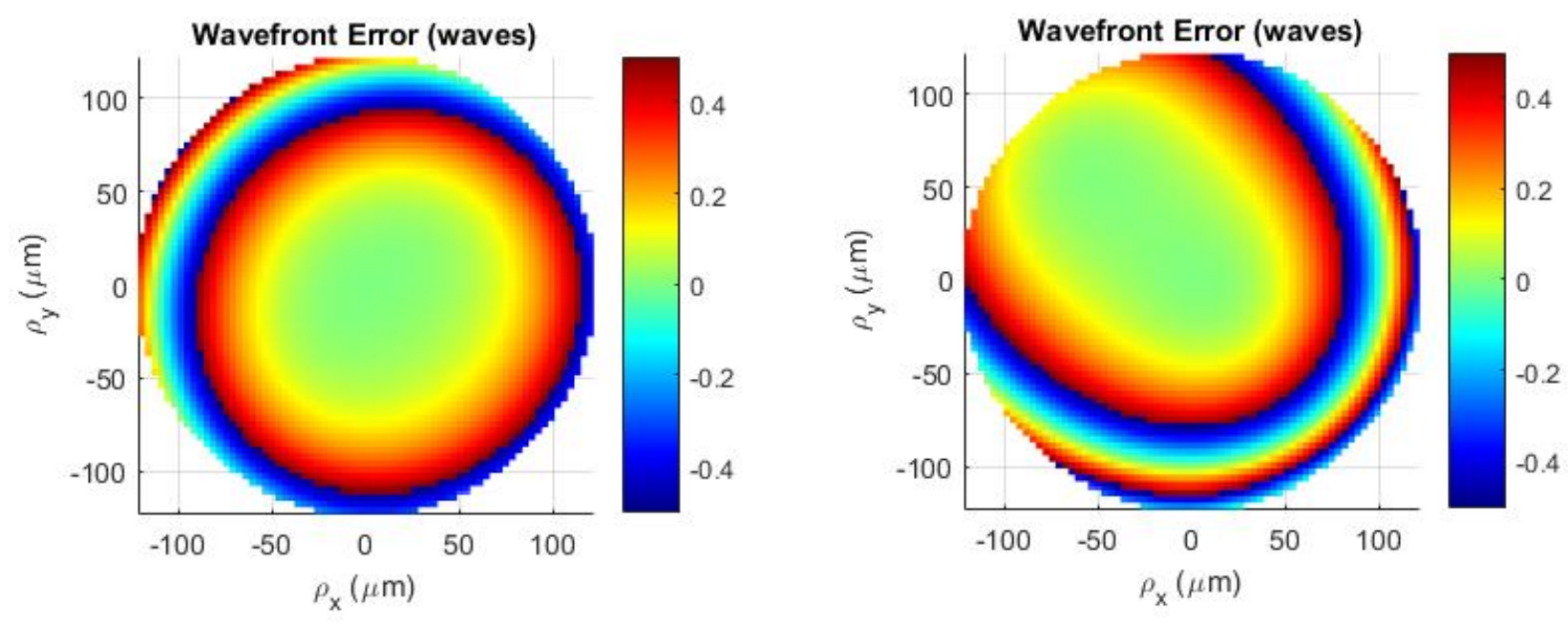

Figure 6: Left: Wavefront aberration field at the exit pupil of the $(-0.25 \mathrm{~mm}, 0.25 \mathrm{~mm})$ decentered channel for on-axis field point $\left(H_{x}=0, H_{y}=0\right)$. Right: Wavefront aberration field at the exit pupil of the $(-0.25 \mathrm{~mm}, 0.25 \mathrm{~mm})$ decentered channel for the normalized field point $\left(H_{x}=-0.707, H_{y}=0.707\right)$.

The wavefront error across the exit pupil of the decentered channel $(-0.25 \mathrm{~mm}, 0.25 \mathrm{~mm})$ is shown in Figure 6 when both on-axis (left) and off-axis (right) field points are applied. In the first case, the microlens displacement perturbs the wavefront map at the Exit Pupil and such a perturbation has the same effect of an off-axis field point applied to the onaxis channel. The wavefront error is induced by the decentered geometry of the system and its aberrations are the same present in an axial sytem when off-axis field points are applied. In the second case, the previous considerations are valid and it can be noted that the wavefront map is determined by the cumulative effects of decentered geometry and off-axis field point. In this example, the wavefront perturbation shown in Figure 6 (right) exhibits the same directionality as the previous example (shown in the right graph in Figure 5) because the microlens displacement and the off-axis field are applied along the same direction and their effects are summed vectorially. The aberrations building the wavefront map are the same as in a rotationally symmetric system, but weighted differently according to the perturbed geometry of the decentered system.

NAT takes into account the modified geometry of a decentered optical system with the definition of an effective field vector $\vec{H}_{e f f}$. For the on-axis channel (Figure 5) the related wavefront error is due to an effective field vector equal to the real field applied to the channel. On the other hand, the wavefront error related to the decentered channel in Figure 6 derives from an effective field vector different from the field really applied $\left(H_{x}=-0.707, H_{y}=0.707\right)$. It is shown that a decentered microlens changes the geometry of an optical channel modifying the dependence of the wavefront error on the applied field points. 
To give more details we consider another channel with a more pronounced decentered coordinate $(3.25 \mathrm{~mm}, 3.25 \mathrm{~mm})$. The wavefront aberration functions at three different field points are shown in the following figures. Figure 7 gives the wavefront functions for normalized field points $\left(H_{x, 1}=0.525, H_{y, 1}=0.531\right), \quad\left(H_{x, 2}=0.603, H_{y, 2}=0.611\right)$ and $\left(H_{x, 3}=0.707, H_{y, 3}=0.710\right)$.
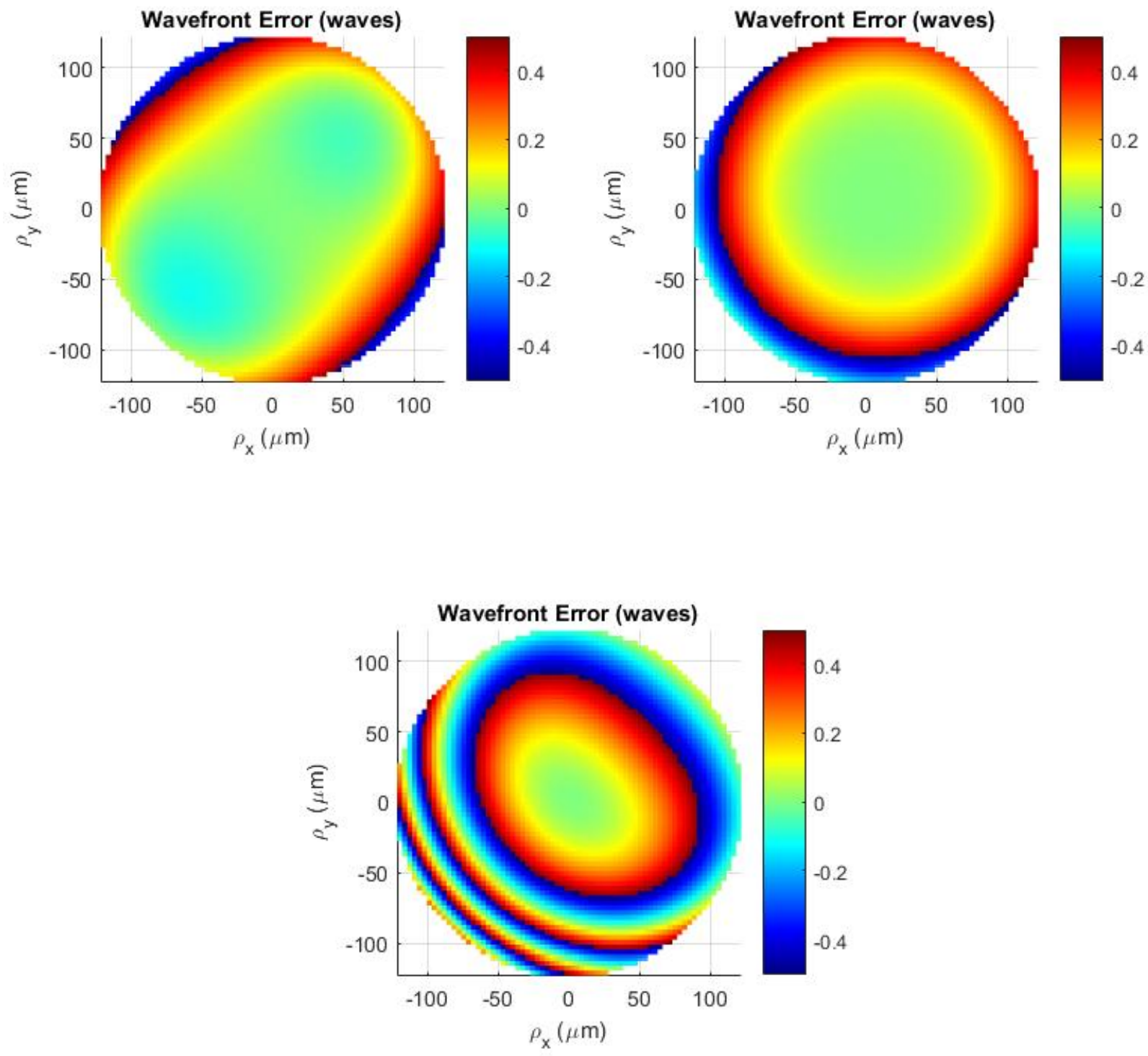

Figure 7: Top Left: Wavefront aberration field at the exit pupil of the $(3.25 \mathrm{~mm}, 3.25 \mathrm{~mm})$ decentered channel for the normalized field point $\left(H_{x, 1}=0.525, H_{y, 1}=0.531\right)$. Top Right: Wavefront aberration field at the exit pupil of the (3.25mm, $3.25 \mathrm{~mm})$ decentered channel for the normalized field point $\left(H_{x, 2}=0.603, H_{y, 2}=0.611\right)$. Bottom: Wavefront aberration

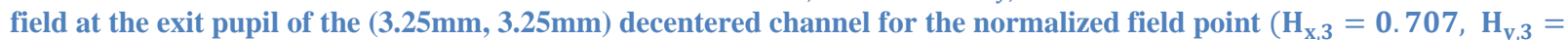
0.710). 
The wavefront functions referred to these three different field points show a particular modulation deriving from the combined effects of geometrical displacement of the microlens and the applied field points. The effective field points processed by a decentered channel are different from the applied field points because of the perturbative effect of decentering on the geometry of an optical channel. The geometrical perturbation (microlens displacement) introduces nodes in the field dependence of the aberration function. The optical channels nodal behavior varies across the MLA plane according to the channels geometrical decentering. Such a nodal behavior becomes clear if the wavefront aberration function is represented as sum of different aberration contributions with the Zernike Fringe decomposition technique.

\section{WAVEFRONT ABERRATION DECOMPOSITION THROUGH ZERNIKE POLYNOMIALS EXPANSION}

A more detailed analysis uses Zernike Fringe polynomials and their dependence on the field to display the aforementioned nodal behavior. The wavefront function at the exit pupil of an optical channel is thus decomposed as sum of Zernike Fringe polynomials weighted by proper coefficients for on-axis as well as for off-axis field points for $\lambda=0.55 \mu m$.

The complex (or generalized) pupil function at the exit pupil of a single-channel optical system is described as

$$
P(x, y)=|P(x, y)| e^{i k W(x, y)}
$$

where $|P(x, y)|$ defines the transmission, size and shape of the exit pupil with the following expression

$$
|P(x, y)|=\left\{\begin{array}{l}
1, \sqrt{x^{2}+y^{2}} \leq 1 \\
0, \sqrt{x^{2}+y^{2}}>1
\end{array}\right\}
$$

and $W(x, y)$ is the Wavefront aberrations function in Cartesian coordinates introducing a phase contribution to the complex pupil function. Using polar coordinates, the wavefront aberration function can be described as follows:

$$
W(\rho, \vartheta)=\sum_{j} \sum_{k} W_{j k} Z_{j k}(\rho, \vartheta)
$$

where $j$ is the index of the surfaces in an optical channel, $k$ is the number of polynomials used in the approximation, $Z_{j k}$ is a single mode in the Zernike Fringe expansion and $W_{j k}$ represents both the coefficient of the Zernike mode and the RMS Wavefront error for a particular mode. From the Zernike polynomials decomposition it is possible to find the contribution of individual aberrations to the wavefront field through the values of the coefficients. 


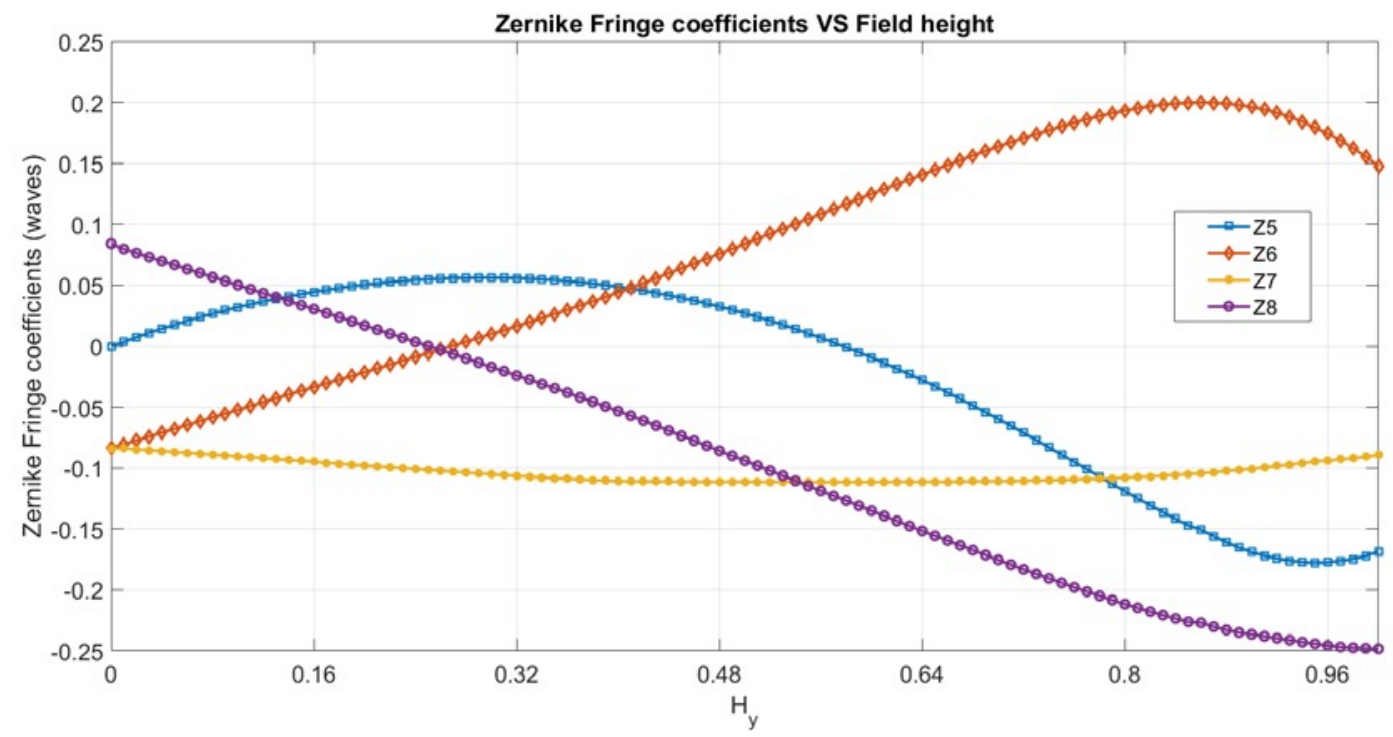

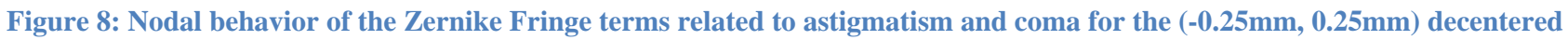
channel.

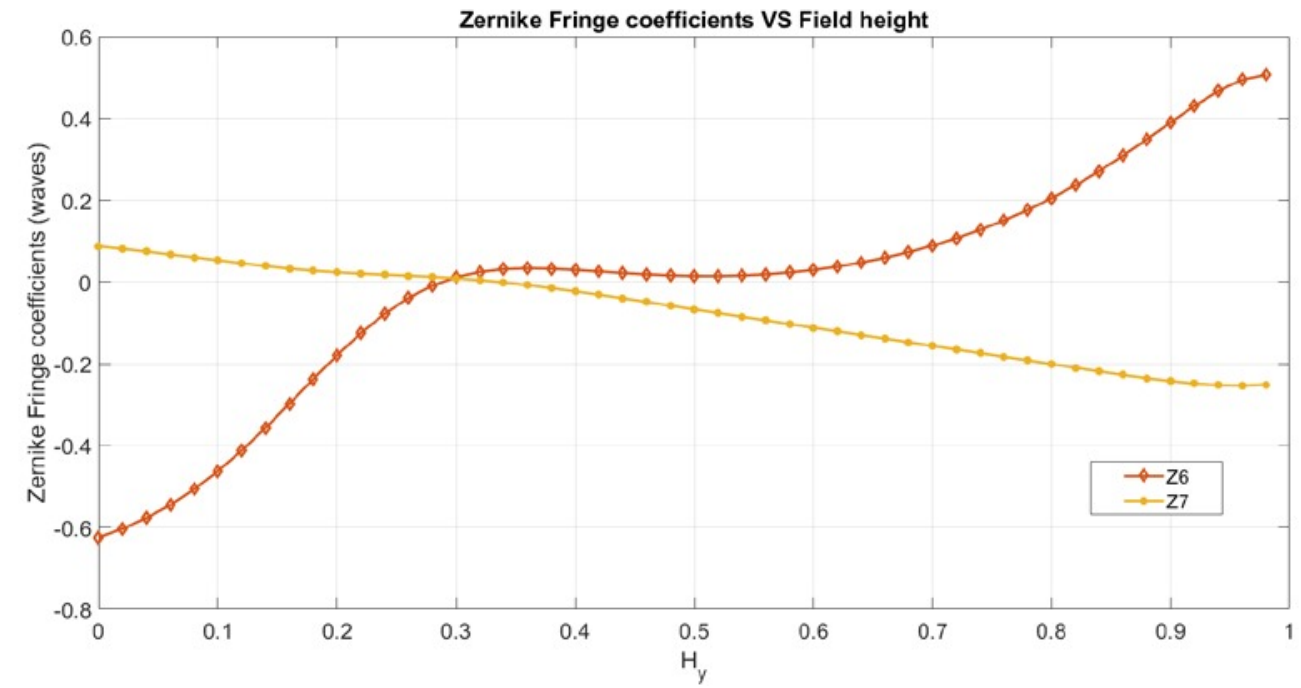

Figure 9: Nodal behavior of the Zernike Fringe terms related to astigmatism and coma for the (3.25mm, 3.25mm) decentered channel.

In Figure 8 the field dependence of the Zernike coefficients is shown for the $(-0.25 \mathrm{~mm}, 0.25 \mathrm{~mm})$ decentered channel. The $5^{\text {th }}$ and $6^{\text {th }}$ Zernike coefficients represent the astigmatism and the $7^{\text {th }}$ and $8^{\text {th }}$ terms are responsible for coma. In a rotationally symmetric optical system, coma has a linear dependence with the field and is zero only for on-axis field points. In a decentered channel, the coma-free field point is shifted along the direction of displacement of the microlens constituting the channel and its position is dependent on the displacement vector $\vec{\sigma}$. In Figure 9 the Zernike Fringe coefficients dependence on the field is shown for the $(3.25 \mathrm{~mm}, 3.25 \mathrm{~mm})$ decentered channel. The $6^{\text {th }}$ Zernike coefficient has a particular dependence on the field known as binodal astigmatism. NAT describes this particular nodal behavior as a consequence of the geometrical perturbation of an optical system that has not been previously corrected for astigmatism. 
The $7^{\text {th }}$ Zernike Fringe coefficient exhibits a node in a shifted field point because of the geometrical displacement of the microlens.

\section{CONCLUSION}

The nodal behavior of the wavefront aberration field in decentered channels of a Focused Plenoptic Camera has been demonstrated. This behavior is a consequence of the loss of rotational symmetry due to decentered microlens in the MLA plane. The displacement of a microlens from the mechanical axis of the camera can be interpreted as a geometrical perturbation to axial symmetry generating a particular modulation of the wavefront aberration field. The wavefront field decomposition with Zernike polynomials shows the new behavior of the aberrations dependence on the field. This fundamental behavior has to be taken into account in the design phase of a Focused Plenoptic Camera.

\section{ACKNOWLEDGEMENTS}

This project has received funding from the European Union's Horizon 2020 research and innovation program under the Marie Sklodowska-Curie grant agreement No 675745 NOLOSS.

\section{REFERENCES}

[1] Ng, R., Levoy, M., Bredif, M., Duval, G., Horowitz, M., and Hanrahan, P., "Light field photography with a handheld plenoptic camera,” Tech. Rep. CTSR 2005-02, Stanford University (2005).

[2] Lumsdaine, A. and Georgiev, T., "The focused plenoptic camera,” in [International Conference on Computational Photography], (April 2009).

[3] C. Perwass and L. Wietzke, “Single-lens 3D camera with extended depth-of-field,” Proc. SPIE 8291, 829108 (2012).

[4] R. V. Shack and K. P. Thompson, "Influence of alignment errors of a telescope system,” Proc. SPIE 251, 146-153 (1980).

[5] K. P. Thompson, "Description of the third-order optical aberrations of near-circular pupil optical systems without symmetry,” J. Opt. Soc. Am. A 22, 1389-1401 (2005).

[6] H. H. Hopkins, The Wave Theory of Aberrations (Clarendon Press, 1950). 